\title{
Composição bromatológica de plantas da Caatinga do Estado da Paraíba, Nordeste do Brasil
}

\section{Homero Perazzo Barbosa ${ }^{1}$, Carolina Uchôa Guerra Barbosa de Lima1, Eduardo Uchôa Guerra Barbosa ${ }^{2}$, , Reinaldo Farias Paiva de Lucena ${ }^{2}$, Elson Soares dos Santos $^{3}$ e Karla Renata Freire Meira ${ }^{4}$}

\author{
${ }^{1}$ Faculdades Nova Esperança. Av. Frei Galvão, 12. Gramame. João Pessoa-PB, Brasil \\ (CEP 58067-695). \\ ${ }^{2}$ Programa de Pós-Graduação em Desenvolvimento e Meio Ambiente. Centro de \\ Ciências Exatas e da Natureza. Universidade Federal da Paraíba. Campus \\ Universitário. João Pessoa-PB, Brasil (CEP 58051-900). *E-mail: \\ eduardouchoa@hotmail.com. \\ ${ }^{3}$ Empresa de Pesquisa Agropecuária da Paraíba - EMEPA. Rodovia PB-008. Costa \\ do Sol. João Pessoa-PB, Brasil (CEP 58033-455). \\ ${ }^{4}$ Universidade Federal da Paraíba. Campus Universitário. João Pessoa-PB, Brasil \\ (CEP 58051-900).
}

Resumo. Considerando-se a importância da diversidade de alimentos disponíveis para os ruminantes na Caatinga do Estado da Paraíba, objetivou-se realizar avaliar o valor nutricional de plantas das espécies estudadas. Foram registradas 176 espécies distribuídas em 125 gêneros e 36 famílias. As famílias mais representativas foram Poaceae (47), Fabaceae (49), Euphorbiaceae (9), Cactaceae (8), Malvaceae (6), Bromeliaceae (5), Convolvulaceae, Amaranthaceae, Anacardiaceae e Rubiaceae (4), Capparaceae, Combretaceae e Cucurbitaceae (3), Agavaceae, Asteraceae, Bignoneaceae e Solonaceae (2). As demais famílias contribuíram com um único representante. Após a identificação, as partes aéreas foram coletadas para as análises bromatológicas. Os principais gêneros em número de espécies foram Urochloa, Cenchrus e Mimosa. Os teores médios (\%) de matéria seca (MS), proteína bruta, fibra bruta e matéria orgânica foram, respectivamente, $28,16 \pm 15,25,3,50 \pm 2,19,7,29 \pm 5,61$ e $26,08 \pm 15,23$. 0 estudo revela uma elevada riqueza de espécies vegetais.

Palavras-chave: Composição química; Composição bromatológica; Ruminantes; Paraíba.

Abstract. Floristic diversity and bromatologic composition of plants from the Paraiba State, Northeast Brazil. Considering the importance of the available food diversity for ruminants in the Caatinga in the Paraíba State, Brazil, the objective of this research was to evaluate the nutritional value of the studied
Recebido:

26/03/2019

Aceito:

24/12/2019

Publicado:

$31 / 12 / 2019$

Acesso aberto

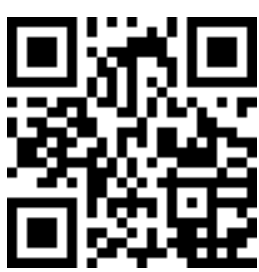

ORCID

(D) $0000-0003-2976-4066$

Homero Perazzo

Barbosa

D 0000-0001-9703-3156

Carolina Uchôa Guerra

Barbosa de Lima 
species. It has been registered 176 species distributed in 125 genera and 36 families. The ones most represented were Poaceae (47), Fabaceae (49), Euphorbiaceae (9), Cactaceae (8), Malvaceae (6), Bromeliaceae (5), Convolvulaceae, Amaranthaceae, Anacardiaceae and Rubiaceae (4), Capparaceae, Combretaceae and Cucurbitaceae (3), Agavaceae, Asteraceae, Bignoneaceae and Solonaceae (2). The remaining families studied contributed with only one species. After the identification, the aerial parts were collected for the bromatologic analyzes. The main genera in number of species were Urochloa, Cenchrus and Mimosa. The average contents (\%) of dry matter (DM), crude protein, crude fiber and organic matter were, respectively, $28.16 \pm 15.25$, $3.50 \pm 2.19,7.29 \pm 5.61$, and $26.08 \pm 15.23$. This study shows a great richness of vegetal species.

Keyword: Chemical composition; Bromatological composition; Ruminants; Paraíba State.

\section{Introdução}

O Estado da Paraíba está localizado na porção oriental da Região Nordeste do Brasil, tendo como área total $56.468,435 \mathrm{~km}^{2}$, com uma população estimada, em 2017, de 4.025.558 habitantes, e uma densidade demográfica de 70,29 hab. $/ \mathrm{km}^{2}$, totalizando 223 municípios (IBGE, 2017).

A vegetação predominante na Caatinga é composta por espécies arbustivas, arbóreas e herbáceas, constituindo uma importante fonte de alimentação para os animais (Andrade et al., 2006). Santos et al. (2010) citam que as plantas xerófilas e caducifólias perdem as folhas com a chegada da estação seca. Algumas espécies botânicas de plantas anuais, cactáceas e bromélias também compõem este bioma. De acordo com Araújo Filho et al. (2002), a folhagem dos arbustos lenhosos em pastagens nativas tem grande importância, uma vez que, representa a maior parte do material disponível, quando as condições ambientais são desfavoráveis.

0 valor nutritivo da forragem é decorrente da concentração de nutrientes, da digestibilidade dos nutrientes e da natureza dos produtos digeridos. Desta forma, o valor nutritivo deve se referir às características inerentes à forragem consumida, às quais deter-
D 0000-0002-6818-7604

Eduardo Uchôa Guerra Barbosa

D 0000-0003-4775-7775

Reinaldo Farias Paiva

de Lucena

D 0000-0002-4293-1124

Elson Soares dos

Santos

(D) 0000-0002-7514-1786

Karla Renata Freire

Meira minam a concentração de energia metabolizável, bem como a eficiência da utilização parcial (Mott e Moore, 1970).

o conhecimento da composição bromatológica é o ponto de partida para o discernimento da concentração e disponibilidade dos nutrientes, o que contribui para predizer a resposta animal em diferentes situações de pastejo (Van Soest, 1994). Neste sentido, a bromatologia consiste em estudar os alimentos, as características de sua composição química e as modificações de seus componentes durante o processamento (Martins, 2007).

Diversas pesquisas indicam que mais de $70 \%$ das plantas da Caatinga compõem, de forma significativa, a dieta dos ruminantes domésticos. Na estação das chuvas, a maior parte da forragem é proporcionada pelo estrato herbáceo, com baixa participação da folhagem de árvores e arbustos. No entanto, à medida que a estação seca se pronuncia, a folhagem das espécies lenhosas passa a constituir a principal fonte de alimento para os animais.

A análise química da forragem fornece informações importantes, que podem promover melhor entendimento dos fatores que limitam o desempenho dos animais ruminantes. No entanto, os métodos de caracterização química não podem estimar diretamente o valor 
nutritivo da forragem, mas sem dúvida, apresentam uma relação direta com a ingestão e com a digestibilidade (Cherney, 2000).

Bakke et al. (2010) ressaltaram a importância de estudos para avaliar espécies arbóreo-arbustivas na alimentação de pequenos ruminantes.

Neste contexto, deve-se propor para a região a adoção de sistemas de produção que gerem competitividade e sustentabilidade para a atividade pecuária, objetivando melhorar os índices produtivos e consequentemente aumentar o retorno econômico para os produtores. Segundo Santos et al. (2010) o cultivo de espécies nativas da Caatinga constitui importante alternativa para aumentar o suprimento de alimentos, principalmente devido a grande adaptabilidade dessas plantas ao ecossistema. Além disso, Voltolini et al. (2010) cita que as pesquisas revelam que mais de $70 \%$ das espécies vegetais da Caatinga participam significativamente da composição da dieta dos animais ruminantes.

Apesar do Estado da Paraíba apresentar boa disponibilidade de fitomassa, parte significativa deste material não é utilizada na alimentação animal, devido ao pouco conhecimento do seu valor nutritivo. Evidencia-se então a necessidade da geração de informações regionalizadas de composição de alimentos permitindo, assim, a melhor utilização dos recursos disponíveis. Isto se torna ainda mais premente diante da complexidade e diversidade de recursos forrageiros encontrados.

Atualmente, entre outros aspectos, tem-se evidenciado a necessidade de estudos sobre o valor nutritivo dessas plantas como fonte de alimentos para os animais.

Considerando-se que as muitas famílias botânicas apresentam grande potencial de utilização na alimentação dos animais, este artigo objetiva identificar espécies do estrato herbáceo, arbustivo e arbóreo além de caracterizá-las bromatologicamente para obtenção de informações do seu valor nutritivo, bus- cando-se um estudo aprofundado para incrementar a produção pecuária.

\section{Material e métodos}

Época e local de coleta

Coletas botânicas mensais, entre julho de 2017 e julho de 2018, nos Municípios de Alagoa Grande, Areia, Campina Grande, Esperança, Patos, Poçinhos, Remígio, São João do Cariri e Soledade, nas estações seca e chuvosa, foram realizadas utilizando os métodos usuais em taxonomia (Judd et al., 2009).

\section{coletadas}

\section{Identificação das espécies}

A identificação das espécies e das famílias foi confirmada com o material botânico depositado no Herbário Lauro Pires Xavier (JPB), da Universidade Federal da Paraíba.

Para efeito de comparação dos compostos bromatológicos, as espécies foram divididas em três grandes grupos compostos pelas Fabaceae, Poaceae e outras famílias.

\section{Análise bromatológica}

Para a análise bromatológica as amostras foram pesadas e submetidas à pré-secagem em estufa de ventilação forçada a $55{ }^{\circ} \mathrm{C}$, por $72 \mathrm{~h}$. Após este procedimento, o material coletado (parte consumida pelos animais ruminantes) foi triturado em moinho marca Willey com peneira de $1 \mathrm{~mm}$ de crivo e acondicionado em recipientes devidamente etiquetados.

As amostras preparadas foram analisadas quanto aos teores de matéria seca (MS), proteína bruta (PB), fibra bruta (FB) e matéria orgânica (MO) segundo metodologias de Silva e Queiroz (2002).

\section{As análises estatísticas}

As análises estatísticas foram realizadas de acordo com o SAS SYSTEM (2002), onde as médias foram comparadas pelo Teste de t de Student a $5 \%$ de probabilidade. 
Tabela 1. Resumo da análise de variância (quadrados médios e significância) para as variáveis MS, PB, FB e MO de plantas de três famílias distintas.

\begin{tabular}{|l|c|c|c|c|c|}
\hline \multirow{2}{*}{ Fontes de variação } & \multirow{2}{*}{ GL } & \multicolumn{4}{|c|}{ Quadrados Médios } \\
\cline { 3 - 6 } & & MS & PB & FB & MO \\
\hline Famílias & 2 & $1007,4736^{*}$ & $96,7581^{* *}$ & $361,1985^{*}$ & $708,0337^{*}$ \\
\hline Erro experimental & 173 & 223,6831 & 3,7274 & 27,7719 & 226,3650 \\
\hline CV $(\%)$ & & 53,11 & 55,23 & 72,29 & 57,69 \\
\hline
\end{tabular}

${ }^{*} \mathrm{e}^{* *}$ Significativo a $5 \%$ e $1 \%$ de probabilidade, respectivamente, pelo teste $\mathrm{F}$.

\section{Resultados e discussão}

As espécies vegetais estudadas apresentam características específicas em função dos diferentes estratos vegetativos. Foram registradas 176 espécies no levantamento florístico, reunidas em 125 gêneros e 36 famílias.

Tabela 2. Relação das famílias botânicas, composição química e nome científico das espécies.

\begin{tabular}{|c|c|c|c|c|c|c|}
\hline Família & Nome vulgar & $\%$ MS & $\%$ PB & $\%$ FB & $\%$ MO & Nome científico \\
\hline Acanthaceae & Melosa & 12,75 & 1,85 & 2,32 & 11,04 & $\begin{array}{l}\text { Ruellia asperula (Mart. ex Nees) } \\
\text { Lindau }\end{array}$ \\
\hline \multirow[t]{2}{*}{ Agavaceae } & Agave & 13,47 & 0,84 & 5,26 & 12,24 & Agave sisalana Perrine \\
\hline & Henequén & 13,26 & 0,4 & 3,42 & 11,74 & Agave fourcroydes Lem. \\
\hline Asparagaceae & Piteira & 14,36 & 1,17 & 4,38 & 12,34 & Furcraea foetida (L.) Haw. \\
\hline \multirow[t]{5}{*}{ Bromeliaceae } & Abacaxi & 12,03 & 0,78 & 2,59 & 11,58 & Ananas comosus (L.) Merril \\
\hline & Gravatá & 5,66 & 0,49 & 1,72 & 4,93 & Bromelia karatas L. \\
\hline & Macambira & 14,44 & 0,83 & 4,78 & 13,48 & $\begin{array}{l}\text { Bromelia laciniosa } \\
\text { Schult. \& Schult.f. }\end{array}$ \\
\hline & Caroá & 11,76 & 0,63 & 4,27 & 10,87 & $\begin{array}{l}\text { Neoglaziovia variegata (Arruda) } \\
\text { Mez }\end{array}$ \\
\hline & $\begin{array}{l}\text { Macambira de } \\
\text { pedra }\end{array}$ & 18,20 & 0,80 & 5,35 & 17,38 & $\begin{array}{l}\text { Encholirium spectabile Mart. ex } \\
\text { Schult. \& Schult.f. }\end{array}$ \\
\hline \multirow[t]{3}{*}{ Capparaceae } & Feijão bravo & 52,36 & 6,63 & 18,8 & 48,14 & Cynophalla flexuosa (L.) J. Presl \\
\hline & Trapiá & 22,65 & 4,50 & 3,38 & 18,98 & Crateva tapia L. \\
\hline & Mussambê & 31,52 & 4,88 & 7,79 & 28,18 & Cleome spinosa Jacq. \\
\hline Pontederiaceae & Aguapé & 11,28 & 0,87 & 2,24 & 9,57 & $\begin{array}{l}\text { Eichhornia crassipes (Mart.) } \\
\text { Solms }\end{array}$ \\
\hline Araceae & Taioba & 8,15 & 1,98 & 1,46 & 8,15 & $\begin{array}{l}\text { Xanthosoma sagittifolium (L.) } \\
\text { Schott }\end{array}$ \\
\hline \multirow[t]{6}{*}{ Malvaceae } & Algodoeiro & 31,75 & 4,36 & 4,25 & 28,55 & Gossypium hirsutumL. \\
\hline & Malva branca & 31,26 & 4,72 & 14,25 & 27,86 & Sida cordifolia L. \\
\hline & Malva roxa & 36,11 & 5,92 & 8,74 & 33,17 & Urena lobata L. \\
\hline & Mela bode & 19,00 & 3,70 & 3,53 & 17,30 & $\begin{array}{l}\text { Herissantia tiubae (K.Schum.) } \\
\text { Brizicky }\end{array}$ \\
\hline & Relógio & 28,36 & 3,60 & 7,44 & 25,3 & Sida rhombifolia L. \\
\hline & Relógio branco & 42,05 & 6,97 & 10,65 & 37,29 & Sida acuta Burm. f. \\
\hline Lecythidaceae & Sapucaia & 33,22 & 4,33 & 2,03 & 31,87 & Lecythis pisonis Cambess. \\
\hline
\end{tabular}


Tabela 2. Continuação.

\begin{tabular}{|c|c|c|c|c|c|c|}
\hline Família & Nome vulgar & $\%$ MS & \% PB & $\%$ FB & $\%$ MO & Nome científico \\
\hline \multirow[t]{4}{*}{ Convolvulaceae } & Amarra cachorro & 15,73 & 2,27 & 5,27 & 14,51 & corymbulosa \\
\hline & Batata doce & 8,54 & 2,49 & 1,28 & 6,99 & Ipomoea batatas (L.) Lam. \\
\hline & Jitirana lisa & 11,50 & 1,78 & 3,07 & 10,71 & $\begin{array}{l}\text { Distimake macrocalyx (Ruiz \& } \\
\text { Pav.) A.R. Simões \& Staples }\end{array}$ \\
\hline & Salsa & 13,14 & 2,36 & 2,00 & 11,82 & $\begin{array}{l}\text { Ipomoea asarifolia (Desr.) Roem. } \\
\& \text { Schult. }\end{array}$ \\
\hline \multirow[t]{4}{*}{ Anacardiaceae } & Umbuzeiro & 19,38 & 2,53 & 2,53 & 17,65 & Spondias tuberosa Arruda \\
\hline & Mangueira & 40,76 & 3,07 & 10,76 & 36,43 & Mangifera indica $\mathrm{L}$. \\
\hline & Aroeira & 46,51 & 5,46 & 5,71 & 44,38 & Schinus terebinthifolia Raddi \\
\hline & Cajueiro & 41,94 & 4,17 & 11,14 & 40,23 & Anacardium occidentale L. \\
\hline \multirow[t]{9}{*}{ Euphorbiaceae } & Aveloz & 14,53 & 0,75 & 3,48 & 12,76 & Euphorbia tirucalli L. \\
\hline & Faveleiro & 18,56 & 4,65 & 2,59 & 16,94 & Cnidoscolus quercifolius Pohl \\
\hline & Velame & 52,05 & 7,74 & 11,56 & 51,47 & Croton campestris A.St.-Hil. \\
\hline & Mamona & 22,48 & 5,54 & 1,55 & 19,89 & Ricinus communis $\mathrm{L}$. \\
\hline & Mandioca & 19,55 & 5,59 & 2,91 & 18,26 & Manihot esculenta Crantz \\
\hline & Maniçoba & 24,16 & 5,34 & 2,94 & 21,56 & Manihot glaziovii Müll.Arg. \\
\hline & Marmeleiro & 39,15 & 7,12 & 4,14 & 35,16 & Croton jacobinensis Baill. \\
\hline & $\begin{array}{l}\text { Marmeleiro } \\
\text { branco }\end{array}$ & 25,64 & 3,19 & 5,39 & 23,58 & Croton sincorensis Mart. \\
\hline & Quebra faca & 64,79 & 7,66 & 8,49 & 58,26 & Croton conduplicatus Kunth \\
\hline Lamiaceae & Bamburral & 18,67 & 3,74 & 2,82 & 15,92 & $\begin{array}{l}\text { Mesosphaerum suaveolens (L.) } \\
\text { Kuntze }\end{array}$ \\
\hline Musaceae & Bananeira & 13,44 & 1,46 & 4,18 & 12,60 & Musa paradisiaca $\mathrm{L}$. \\
\hline Bombacaceae & Barriguda & 38,10 & 3,42 & 7,01 & 33,70 & Ceiba pentandra (L.) Gaertn. \\
\hline Portulacaceae & Beldroega & 14,92 & 1,66 & 2,26 & 11,48 & Portulaca oleracea L. \\
\hline Celastraceae & Bom nome & 23,40 & 1,67 & 5,65 & 21,84 & Monteverdia rigida (Mart.) Biral \\
\hline Apocynaceae & Pereiro & 19,99 & 1,01 & 3,28 & 15,63 & $\begin{array}{l}\text { Aspidosperma pyrifolium Mart. \& } \\
\text { Zucc. }\end{array}$ \\
\hline Nictaginaceae & Pega pinto & 19,69 & 3,83 & 3,17 & 16,56 & Boerhavia coccinea Mill. \\
\hline \multirow[t]{4}{*}{ Amaranthaceae } & Quebra panela & 36,43 & 2,74 & 13,24 & 32,93 & $\begin{array}{lll}\text { Alternanthera } & \text { brasiliana } \\
\text { Kuntze } & \text { (L.) }\end{array}$ \\
\hline & Ervanço & 25,74 & 1,77 & 9,02 & 22,15 & $\begin{array}{l}\text { Froelichia humboldtiana (Roem. } \\
\text { \& Schult.) Seub. }\end{array}$ \\
\hline & Bredo & 15,10 & 2,36 & 3,34 & 12,23 & Amaranthus viridis L. \\
\hline & Bredo de espinho & 19,72 & 3,13 & 2,77 & 16,91 & Amaranthus spinosus L. \\
\hline \multirow[t]{2}{*}{ Asteraceae } & Camará de bucha & 19,18 & 3,09 & 2,56 & 16,25 & $\begin{array}{l}\text { Verbesina macrophylla (Cass.) } \\
\text { S.F.Blake }\end{array}$ \\
\hline & $\begin{array}{l}\text { Carrapicho de } \\
\text { agulha }\end{array}$ & 11,64 & 2,69 & 1,57 & 86,95 & Bidens cynapiifolia Kunth \\
\hline \multirow[t]{4}{*}{ Rubiaceae } & Vassourinha & 34,97 & 2,42 & 8,92 & 33,56 & Spermacoce latifolia $L$. \\
\hline & $\begin{array}{l}\text { Vassourinha de } \\
\text { botão }\end{array}$ & 22,04 & 2,49 & 5,58 & 20,38 & Spermacoce verticillata $L$. \\
\hline & Fato de piaba & 39,08 & 3,10 & 14,00 & 34,02 & $\begin{array}{l}\text { Richardia grandiflora (Cham. \& } \\
\text { Schltdl.) Steud }\end{array}$ \\
\hline & Mentrasto & 21,28 & 2,21 & 3,79 & 17,22 & Ageratum conyzoides L. \\
\hline Cactaceae & Palmatória & 14,07 & 0,46 & 1,82 & 10,59 & Opuntia monacantha Haw. \\
\hline
\end{tabular}


Tabela 2. Continuação.

\begin{tabular}{|c|c|c|c|c|c|c|}
\hline Família & Nome vulgar & $\% \mathrm{MS}$ & $\%$ PB & $\%$ FB & $\%$ MO & Nome científico \\
\hline \multirow[t]{7}{*}{ Cactaceae } & Cardeiro & 12,91 & 0,94 & 1,62 & 11,36 & $\begin{array}{l}\text { Pilosocereus chrysostele (Voupel) } \\
\text { Byles \& Rowley }\end{array}$ \\
\hline & Xique xique & 10,99 & 0,56 & 2,01 & 9,01 & $\begin{array}{ll}\text { Pilosocereus gounellei } & \text { (F.A.C. } \\
\text { Weber) Byles \& Rowley } & \\
\end{array}$ \\
\hline & Coroa de frade & 11,47 & 0,85 & 2,90 & 9,29 & Melocactus violaceus Pfeiff. \\
\hline & Facheiro & 12,86 & 0,93 & 1,97 & 9,79 & $\begin{array}{l}\text { Facheiroa squamosa (Gürke) } \\
\text { P.J.Braun \& Esteves }\end{array}$ \\
\hline & Palma forrageira & 9,63 & 0,57 & 1,21 & 7,47 & Opuntia ficus-indica (L.) Mill. \\
\hline & Palma gigante & 6,77 & 0,59 & 1,56 & 5,55 & Opuntia ficus-indica (L.) Mill. \\
\hline & Palma miúda & 7,07 & 0,79 & 0,76 & 5,13 & $\begin{array}{l}\text { Nopalea cochenillifera (L.) Salm- } \\
\text { Dyck }\end{array}$ \\
\hline Boraginaceae & Fedegoso & 27,10 & 5,16 & 3,35 & 22,06 & Heliotropium indicum L. \\
\hline Myrtaceae & Oliveira & 38,96 & 2,76 & 7,95 & 37,12 & Syzygium cumini (L.) Skeels \\
\hline Moraceae & Jaqueira & 34,73 & 3,12 & 6,14 & 29,77 & Artocarpus heterophyllus Lam. \\
\hline Rhamnaceae & Juazeiro & 46,12 & 5,69 & 12,87 & 42,34 & Ziziphus joazeiro Mart. \\
\hline \multirow[t]{2}{*}{ Solonaceae } & Maria branca & 13,98 & 3,00 & 2,14 & 12,14 & Cestrum mariquitense Kunth \\
\hline & Jurubeba & 22,31 & 5,38 & 3,48 & 20,51 & Solanum paniculatum $\mathrm{L}$. \\
\hline \multirow[t]{3}{*}{ Cucurbitaceae } & Maxixe & 11,28 & 3,03 & 1,42 & 9,39 & Cucumis anguria $\mathrm{L}$. \\
\hline & Melancia & 2,80 & 0,53 & 0,72 & 2,30 & $\begin{array}{ll}\text { Citrullus lanatus (Thunb.) } \\
\text { Matsum. \& Nakai }\end{array}$ \\
\hline & $\begin{array}{l}\text { Melão de São } \\
\text { Caetano }\end{array}$ & 26,02 & 4,98 & 5,77 & 23,61 & Momordica charantia L. \\
\hline Cyperaceae & Minhocão & 35,55 & 3,81 & 11,00 & 31,45 & $\begin{array}{l}\text { Eleocharis elegans (Kunth) } \\
\text { Roem. \& Schult. }\end{array}$ \\
\hline Chrysobalanaceae & Oiticica & 35,65 & 2,81 & 12,26 & 33,59 & Licania rigida Benth. \\
\hline \multirow[t]{2}{*}{ Bignoniaceae } & Pau d'arco & 22,01 & 5,79 & 5,57 & 20,63 & $\begin{array}{l}\text { Tabebuia serratifolia (Vahl) G. } \\
\text { Nicholson }\end{array}$ \\
\hline & Craibeira & 41,11 & 2,94 & 12,76 & 38,26 & $\begin{array}{l}\text { Tabebuia aurea (Silva Manso) } \\
\text { Benth. \& Hook.f. ex S. Moore }\end{array}$ \\
\hline \multirow[t]{3}{*}{ Combretaceae } & Bugi & 24,36 & 7,04 & 5,72 & 22,70 & Combretum laxum Jacq. \\
\hline & Mofumbu & 57,24 & 8,05 & 9,16 & 52,04 & Combretum leprosum Mart. \\
\hline & Sipaúba & 65,9 & 10,41 & 10,33 & 62,74 & Combretum glaucocarpum Mart. \\
\hline Sapotaceae & Rompe gibão & 29,90 & 5,38 & 4,84 & 28,93 & $\begin{array}{l}\text { Sideroxylon obtusifolium (Roem. } \\
\text { \& Schult.) T.D. Penn }\end{array}$ \\
\hline \multirow[t]{9}{*}{ Fabaceae } & Arroz de cabra & 25,88 & 6,24 & 3,03 & 23,99 & Desmanthus virgatus (L.) Willd. \\
\hline & Angico & 35,89 & 7,53 & 13,63 & 34,21 & $\begin{array}{l}\text { Anadenanthera colubrina (Vell.) } \\
\text { Brenan }\end{array}$ \\
\hline & Camunzé & 31,17 & 7,35 & 7,00 & 30,20 & $\begin{array}{l}\text { Pithecellobium polycephalum } \\
\text { Benth. }\end{array}$ \\
\hline & Orelha de onça & 19,32 & 1,54 & 6,87 & 17,55 & $\begin{array}{l}\text { Macroptilium martii (Benth.) } \\
\text { Maréchal \& Baudet }\end{array}$ \\
\hline & Pau brasil & 39,70 & 3,04 & 9,82 & 36,92 & $\begin{array}{l}\text { Paubrasilia echinata (Lam.) } \\
\text { Gagnon, H.C. Lima \& G.P. Lewis }\end{array}$ \\
\hline & $\begin{array}{l}\text { Rapadura de } \\
\text { cavalo }\end{array}$ & 14,79 & 2,81 & 4,45 & 13,66 & Desmodium subsecundum Vogel \\
\hline & Sabiá & 37,89 & 7,04 & 5,77 & 35,62 & Mimosa caesalpiniifolia Benth. \\
\hline & Siratro & 23,11 & 4,15 & 7,13 & 20,54 & $\begin{array}{l}\text { Macroptilium atropurpureum } \\
\text { (Sessé \& Moc. ex DC.) Urb. }\end{array}$ \\
\hline & Sucupira & 18,32 & 4,19 & 3,71 & 17,53 & Bowdichia virgilioides Kunth \\
\hline
\end{tabular}


Tabela 2. Continuação.

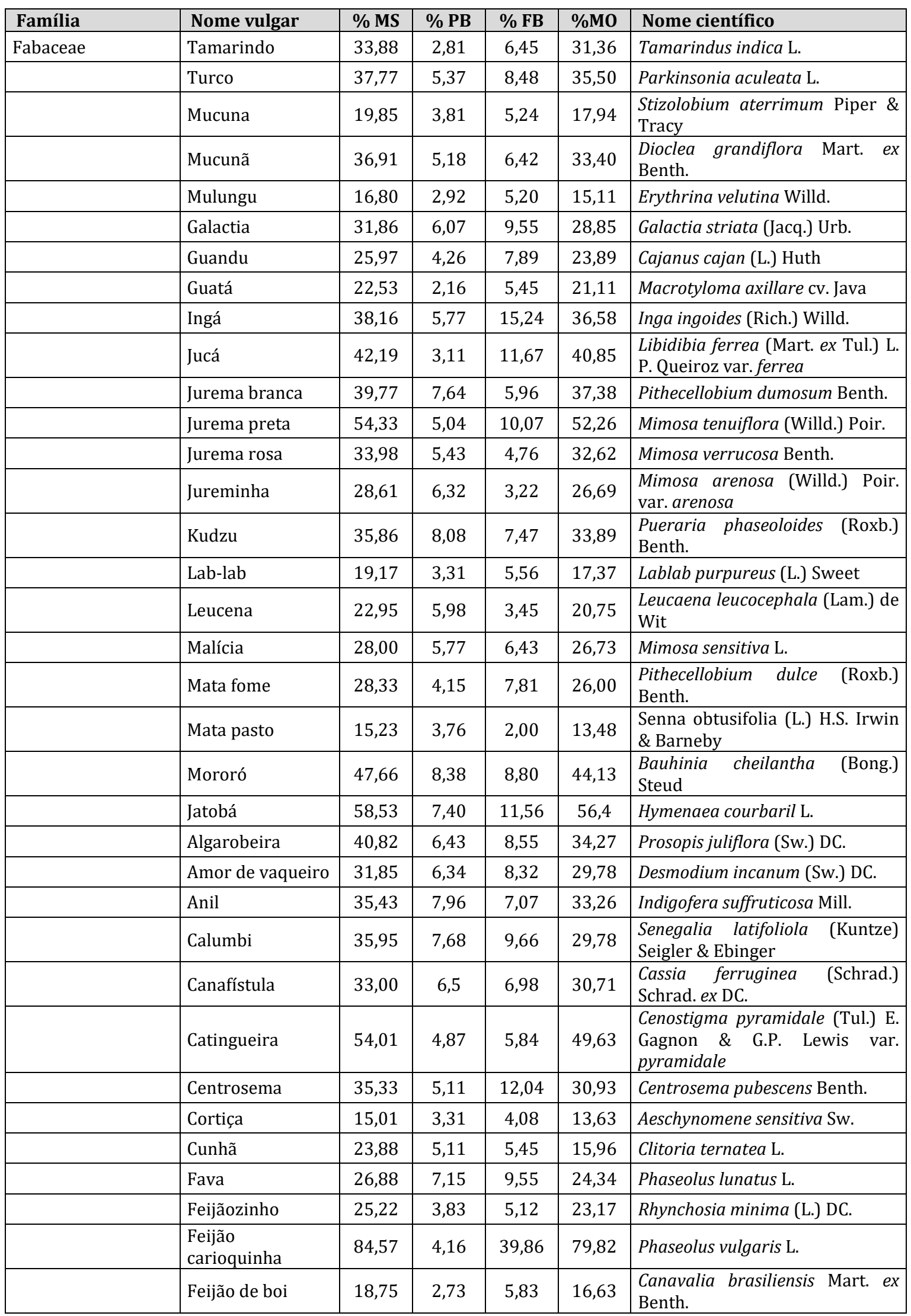


Tabela 2. Continuação.

\begin{tabular}{|c|c|c|c|c|c|c|}
\hline Família & Nome vulgar & $\% \mathrm{MS}$ & $\%$ PB & $\%$ FB & $\%$ MO & Nome científico \\
\hline \multirow[t]{5}{*}{ Fabaceae } & Feijão de porco & 20,34 & 4,82 & 3,77 & 27,52 & Canavalia ensiformis (L.) DC. \\
\hline & Feijão de rola & 35,46 & 4,13 & 12,7 & 32,88 & $\begin{array}{l}\text { Macroptilium gracile (Poepp. ex } \\
\text { Benth.) Urb. }\end{array}$ \\
\hline & Feijão macassar & 12,74 & 3,75 & 1,84 & 11,64 & Vigna unguiculata (L.) Walp. \\
\hline & Feijão mulatinho & 17,17 & 2,77 & 4,71 & 15,54 & Phaseolus vulgaris $\mathrm{L}$. \\
\hline & Calopogônio & 19,65 & 4,52 & 4,8 & 17,33 & Calopogonium mucunoides Desv. \\
\hline \multirow[t]{29}{*}{ Poaceae } & Capim amargoso & 25,19 & 1,95 & 9,32 & 22,69 & Digitaria insularis (L.) Fedde \\
\hline & Cana-de-açúcar & 26,22 & 0,73 & 9,19 & 25,1 & Saccharum officinarum L. \\
\hline & $\begin{array}{l}\text { Capim } \\
\text { andropogon }\end{array}$ & 25,55 & 1,49 & 14,54 & 24,28 & Andropogon gayanus Kunth \\
\hline & $\begin{array}{l}\text { Capim barba de } \\
\text { bode }\end{array}$ & 26,75 & 1,68 & 3,23 & 20,78 & Aristida pallens Cav. \\
\hline & Capim belota & 34,24 & 2,66 & 9,34 & 30,97 & Chloris radiata (L.) Sw. \\
\hline & Capim belota roxo & 37,70 & 1,86 & 13,24 & 34,05 & Chloris barbata Sw. \\
\hline & Capim brizantha & 23,79 & 1,47 & 8,26 & 21,81 & $\begin{array}{l}\text { Urochloa brizantha (Hochst. ex } \\
\text { A. Rich.) R.D. Webster }\end{array}$ \\
\hline & Capim búffel & 31,59 & 1,8 & 10,94 & 28,03 & Cenchrus ciliaris $\mathrm{L}$. \\
\hline & Capim cameron & 15,99 & 2,69 & 6,32 & 14,21 & $\begin{array}{l}\text { Cenchrus purpureus (Schumach.) } \\
\text { Morrone }\end{array}$ \\
\hline & Capim colonião & 23,41 & 3,48 & 7,93 & 21,05 & $\begin{array}{l}\text { Megathyrsus maximus (Jacq.) } \\
\text { B.K. Simon \& S.W.L. Jacobs }\end{array}$ \\
\hline & Capim d’água & 24,15 & 3,19 & 6,45 & 20,53 & $\begin{array}{ll}\text { Paspalidium } & \text { geminatum } \\
\text { (Forssk.) Stapf } & \end{array}$ \\
\hline & Capim de planta & 26,95 & 1,88 & 8,59 & 23,74 & $\begin{array}{l}\text { Urochloa mutica (Forssk.) T.Q. } \\
\text { Nguyen }\end{array}$ \\
\hline & Capim de raiz & 18,82 & 1,60 & 6,33 & 17,18 & Chloris orthonoton Döll \\
\hline & Capim de roça & 87,13 & 5,04 & 35,44 & 83,58 & Digitaria sanguinalis (L.) Scop. \\
\hline & $\begin{array}{l}\text { Capim elefante } \\
\text { napier }\end{array}$ & 19,14 & 1,38 & 7,36 & 17,37 & $\begin{array}{l}\text { Cenchrus purpureus (Schumach.) } \\
\text { Morrone }\end{array}$ \\
\hline & $\begin{array}{l}\text { Capim elefante } \\
\text { roxo }\end{array}$ & 16,33 & 1,39 & 5,04 & 13,9 & $\begin{array}{l}\text { Cenchrus purpureus (Schumach.) } \\
\text { Morrone }\end{array}$ \\
\hline & Capim eragrostis & 41,87 & 5,40 & 12,66 & 39,92 & $\begin{array}{l}\text { Eragrostis cilianensis (All.) } \\
\text { Vignolo ex Janch. }\end{array}$ \\
\hline & Capim estrela & 31,66 & 2,30 & 10,05 & 28,8 & $\begin{array}{l}\text { Cynodon plectostachyus (K. } \\
\text { Schum.) Pilg. }\end{array}$ \\
\hline & Capim forquilha & 32,19 & 1,82 & 7,76 & 25,41 & Paspalum notatum Flüggé \\
\hline & Capim gengibre & 14,84 & 1,25 & 4,92 & 13,67 & Paspalum maritimum Trin. \\
\hline & Capim gordura & 19,01 & 2,38 & 3,34 & 17,43 & Melinis minutiflora P. Beauv. \\
\hline & $\begin{array}{l}\text { Capim grama de } \\
\text { burro }\end{array}$ & 42,40 & 2,14 & 13,65 & 38,84 & Cynodon dactylon (L.) Pers. \\
\hline & Capim gramafante & 25,60 & 1,97 & 8,10 & 21,80 & $\begin{array}{l}\text { Cenchrus purpureus (Schumach.) } \\
\text { Morrone }\end{array}$ \\
\hline & Capim jaraguá & 31,81 & 1,46 & 9,78 & 27,27 & Hyparrhenia rufa (Nees) Stapf \\
\hline & Capim lucas & 30,93 & 2,29 & 9,00 & 29,14 & Sporobolus indicus (L.) R.Br. \\
\hline & Capim mandante & 19,42 & 1,21 & 6,02 & 16,87 & $\begin{array}{l}\text { Echinochloa polystachya (Kunth) } \\
\text { Hitchc. }\end{array}$ \\
\hline & $\begin{array}{l}\text { Capim mão de } \\
\text { sapo }\end{array}$ & 12,93 & 1,43 & 4,12 & 11,19 & $\begin{array}{l}\text { Dactyloctenium aegyptium (L.) } \\
\text { Willd. }\end{array}$ \\
\hline & Capim marreca & 24,31 & 1,12 & 7,59 & 20,84 & $\begin{array}{lll}\begin{array}{l}\text { Paspalum } \\
\text { Bergius }\end{array} & \text { conjugatum } & \text { P.J. } \\
\end{array}$ \\
\hline & Capim milhã & 27,39 & 1,73 & 8,67 & 25,03 & $\begin{array}{l}\text { Urochloa plantaginea (Link) R.D. } \\
\text { Webster }\end{array}$ \\
\hline
\end{tabular}


Tabela 2. Continuação.

\begin{tabular}{|c|c|c|c|c|c|c|}
\hline Família & Nome vulgar & $\%$ MS & $\%$ PB & $\%$ FB & $\%$ MO & Nome científico \\
\hline \multirow[t]{18}{*}{ Poaceae } & $\begin{array}{l}\text { Capim milhã } \\
\text { preta }\end{array}$ & 14,40 & 1,55 & 4,80 & 12,76 & $\begin{array}{l}\text { Paspalum fasciculatum Willd. ex } \\
\text { Flüggé }\end{array}$ \\
\hline & Capim mimoso & 21,05 & 1,09 & 8,16 & 19,28 & Eragrostis ciliaris (L.) R.Br. \\
\hline & Capim orvalho & 78,74 & 3,01 & 26,76 & 75,38 & $\begin{array}{ll}\text { Sporobolus } & \text { tenuissimus } \\
\text { (Schrank) Kuntze } & \\
\end{array}$ \\
\hline & Capim panasco & 70,56 & 2,09 & 18,39 & 63,96 & Eragrostis pilosa (L.) P. Beauv. \\
\hline & Capim pangola & 44,44 & 2,21 & 14,02 & 41,05 & $\begin{array}{l}\text { Digitaria eriantha pentzii (Stent) } \\
\text { Kok }\end{array}$ \\
\hline & $\begin{array}{l}\text { Capim pé de } \\
\text { galinha }\end{array}$ & 27,31 & 2,93 & 8,24 & 24,19 & Eleusine indica (L.) Gaertn. \\
\hline & $\begin{array}{l}\text { Capim pé de } \\
\text { nambu }\end{array}$ & 85,44 & 4,29 & 33,39 & 80,37 & $\begin{array}{lll}\text { Setaria } & \text { parviflora } & \text { (Poir.) } \\
\text { Kerguélen } & & \\
\end{array}$ \\
\hline & Capim sândalo & 31,81 & 1,41 & 11,92 & 29,64 & $\begin{array}{lll}\begin{array}{l}\text { Chrysopogon } \\
\text { Roberty }\end{array} & \text { zizanioides } & \text { (L.) } \\
\end{array}$ \\
\hline & Capim seda & 26,85 & 1,85 & 10,16 & 22,11 & Tricholaena rosea Nees \\
\hline & $\begin{array}{l}\text { Capim sempre } \\
\text { verde }\end{array}$ & 22,96 & 1,71 & 8,18 & 20,52 & $\begin{array}{l}\text { Megathyrsus maximus (Jacq.) } \\
\text { B.K. Simon \& S.W.L.Jacobs }\end{array}$ \\
\hline & $\begin{array}{l}\text { Capim tanner } \\
\text { grass }\end{array}$ & 17,22 & 3,26 & 4,44 & 15,21 & $\begin{array}{l}\text { Urochloa arrecta (Hack. ex T. } \\
\text { Durand \& Schinz) Morrone \& } \\
\text { Zuloaga }\end{array}$ \\
\hline & Capim terra seca & 21,39 & 1,90 & 7,19 & 19,59 & $\begin{array}{l}\text { Urochloa humidicola (Rendle) } \\
\text { Morrone \& Zuloaga }\end{array}$ \\
\hline & Carrapicho & 18,92 & 2,33 & 6,31 & 16,67 & Cenchrus echinatus L. \\
\hline & Capim decumbens & 24,32 & 1,63 & 8,49 & 22,58 & $\begin{array}{l}\text { Urochloa decumbens (Stapf) R.D. } \\
\text { Webster }\end{array}$ \\
\hline & Milho & 23,79 & 0,66 & 8,55 & 23,28 & Zea mays L. \\
\hline & Ruziziensis & 16,74 & 2,11 & 4,89 & 15,13 & $\begin{array}{l}\text { Urochloa ruziziensis (R. Germ. \& } \\
\text { Evrard) Crins }\end{array}$ \\
\hline & Sorgo forrajeiro & 62,77 & 2,16 & 20,32 & 57,75 & Sorghum bicolor (L.) Moench \\
\hline & Bambu & 43,24 & 10,19 & 9,36 & 37,19 & $\begin{array}{l}\text { Bambusa vulgaris Schrad. ex J.C. } \\
\text { Wendl. }\end{array}$ \\
\hline
\end{tabular}

Da avaliação total a Família Fabaceae contribuiu com 27,68\% (49 espécies), a Família Poaceae com 26,55\% (47 espécies) representando as duas famílias $54,23 \%$ das espécies estudadas. 0 componente Outras Famílias contribuiu com 45,76\% sendo representadas por 80 espécies (Figura 1). A presença de Fabaceae em estudos fitossociológicos é comum, pois é a família com grande número de espécies presente em diversos biomas (Giulietti et al., 2005). Pesquisas desenvolvidas no
Nordeste brasileiro evidenciam que $70 \%$ das espécies da Caatinga participam, significativamente, da composição da dieta dos ruminantes (Santos et al., 2005). Santana et al. (2011) destacaram a presença de Euphorbiaceae no componente arbustivo da caatinga.

Outras pesquisas destacaram a presença das famílias, Euphorbiaceae, Fabaceae, Malvaceae e Poaceae como famílias de grande representatividade (Pereira Júnior et al., 2012; Farias et al., 2016). 


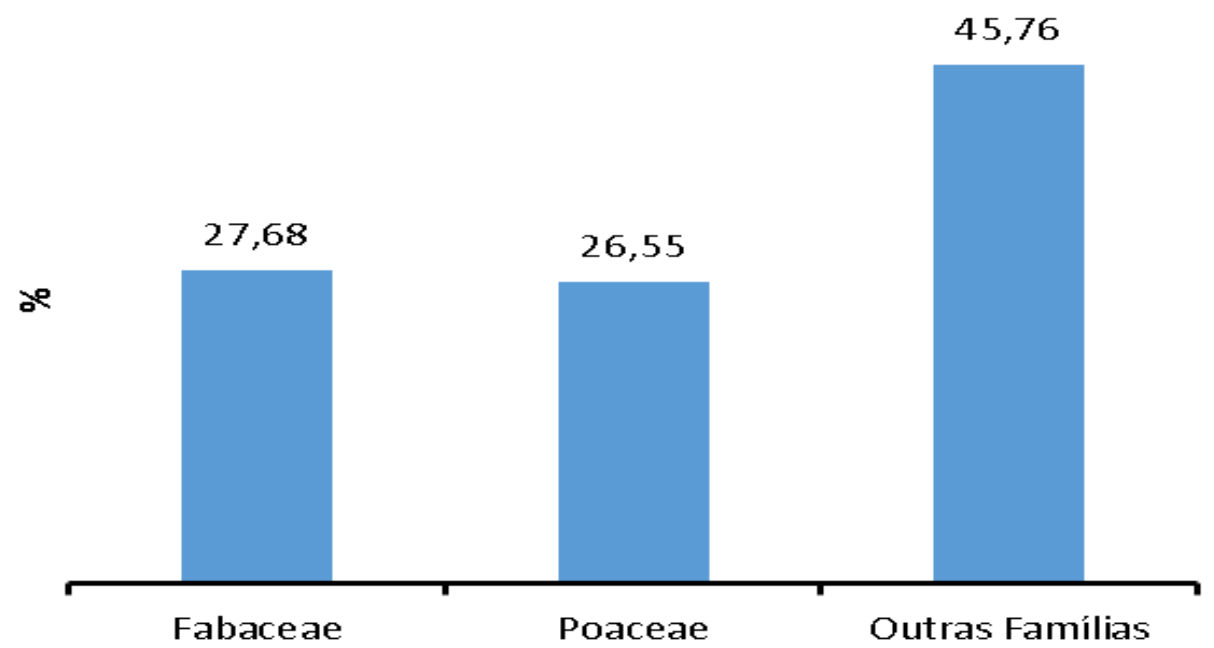

Figura 1. Distribuição das espécies estudadas por famílias.

Foram avaliados os teores de MS, PB, FB e MO. A matéria seca é a porção do alimento onde estão todos os nutrientes, isto é, a massa total sem considerar a umidade. Deve ser usada para expressar a concentração de nutrientes e, a partir daí, determinar o valor nutritivo. De acordo com a Tabela 3 houve diferença significativa $(\mathrm{P}<0,05)$ entre os valores médios de MS, PB, FB e de MO para os grupos de famílias estudadas, cabendo destacar os teores mais altos de PB para a família Fabaceae. Por sua vez, a família Poaceae apresentou maior concentração para a FB.

Tabela 3. Teores médios de matéria seca (MS), de proteína bruta (PB), de fibra bruta (FB) e de matéria orgânica (MO) das famílias estudadas (Dados em \% e expressos na matéria natural).

\begin{tabular}{|l|c|c|c|c|c|}
\hline \multicolumn{1}{|c|}{ Famílias } & $\mathbf{N}$ & MS & PB & FB & MO \\
\hline Fabaceae & 49 & $31,23^{\mathrm{a}}$ & $5,06^{\mathrm{a}}$ & $7,68^{\mathrm{b}}$ & $28,97^{\mathrm{a}}$ \\
\hline Poaceae & 47 & $31,26^{\mathrm{a}}$ & $2,28^{\mathrm{c}}$ & $10,23^{\mathrm{a}}$ & $28,34^{\mathrm{ab}}$ \\
\hline Outras Famílias & 80 & $24,45^{\mathrm{b}}$ & $3,25^{\mathrm{b}}$ & $5,32^{\mathrm{c}}$ & $22,98^{\mathrm{b}}$ \\
\hline
\end{tabular}

Médias seguidas da mesma letra, nas colunas, não diferem entre pelo teste $t$ de Student a 5\% de probabilidade.

Os teores de MS apresentaram diferença significativa $(\mathrm{P}<0,05)$ entre os grupos de família estudados, com valores extremos observados para Araceae $(8,15 \%)$ e Combretaceae $(49,17 \%)$. 0 teor médio de MS das espécies estudadas foi de 28,16\%, valor inferior ao encontrado por Santana et al. (2011) de $55,79 \%$.
Os compostos nitrogenados proteicos e não proteicos aparecem, no resultado da análise bromatológica, como proteína bruta. Esta é determinada medindo-se o total de nitrogênio $(\mathrm{N})$ e multiplicando-se por 6,25 (as proteínas têm em média $16 \%$ de $\mathrm{N}$ no aminoácido). $A$ adição de ureia na silagem aumenta o teor de N, mas não de proteína verdadeira (Queiroz et al., 2000). 
Grande parte da PB em forragens pode ser nitrogênio não-proteico (NNP). O NNP é assumido como completamente degradável no rúmen, servindo para atender parte da demanda de $\mathrm{N}$ necessária para síntese de proteína microbiana. O NNP supre parte da demanda nutricional de proteína degradável no rúmen (PDR). Os microrganismos do rúmen são a fonte, quantitativamente mais importante, de aminoácidos no intestino dos ruminantes (Queiroz et al., 2000).

Os menores teores de proteína bruta (PB) foram observados para as famílias Bromeliaceae e Cataceae com $0,71 \%$. A Combretaceae se destacou entre todas apresentando o valor de $8,50 \%$, o mais elevado entre as famílias sendo seguida pela Fabaceae $(5,06 \%)$. A média de proteína bruta das espécies estudadas foi de $3,50 \% \pm 2,19$.

$O$ valor médio de $P B$, expresso na matéria seca, observado nessa pesquisa, foi de $12,43 \%$, valor superior a $7,00 \%$ de PB que, para dietas dos ruminantes, é considerado por Minson et al. (1976) como sendo o valor mínimo para que ocorra adequado crescimento da microflora microbiana e com isso que os animais alcancem níveis de consumo e digestibilidade suficientes para sua manutenção.

Moreira et al. (2006), estudando a composição química de várias espécies arbóreas, arbustivas e herbáceas da Caatinga, na época chuvosa do ano, encontraram teores médios de PB que variaram de 7,61 a 16,88\%. Santana et al. (2011) encontraram teor médio de PB de $15,5 \%$.
A fibra é a fração dos carboidratos que é usada como fonte energética pelos microrganismos do rúmen. Tem sido usada para caracterizar os alimentos e para estabelecer limites de utilização dos ingredientes nas rações (VAN SOEST, 1994). No entanto, as pesquisas realizadas não chegaram a um consenso sobre a concentração ideal de fibra para a otimização do consumo de energia por ruminantes (MERTENS et al., 1994). A fibra é essencial, uma vez que os ácidos graxos voláteis (AGV) produzidos durante a fermentação ruminal constituem a principal fonte de energia para o animal (MERTENS, 2001).

Para fibra bruta (FB) os valores variaram de 1,46\% (Araceae) a 12,87\% (Rhamnaceae). A média de FB das espécies estudadas foi de 7,29\% $\pm 5,61$.

A matéria orgânica é a parte do alimento sem serem consideradas as cinzas (matéria mineral). Os valores médios de MO variaram de 8,15\% (Araceae) a 45,83\% (Combretaceae) com média, para as famílias estudadas de $26,08 \% \pm 15,23$. Valores semelhantes foram determinados por Araújo et al. (2006) ao analisarem forrageiras arbustivas e arbóreas na região semiárida.

Os teores médios de MS, PB, FB e de MO foram, respectivamente de $31,23 \%, 5,06 \%, 7,68 \%$ e de $28,97 \%$ para Fabaceae, de 31,26\%, 2,28\%, 10,23\% e de $28,34 \%$ para Poaceae e de $24,45 \%$, $3,25 \%, 5,32 \%$ e de $22,98 \%$ para as demais famílias estudadas (Figuras 2 a 5). 


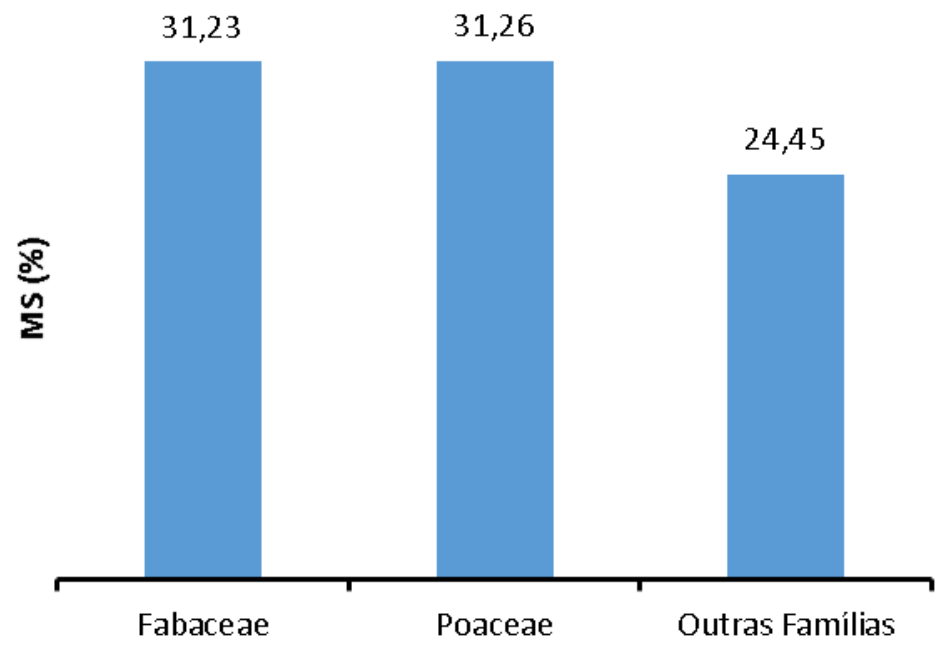

Figura 2. Teor médio de matéria seca (MS) para as famílias estudadas.

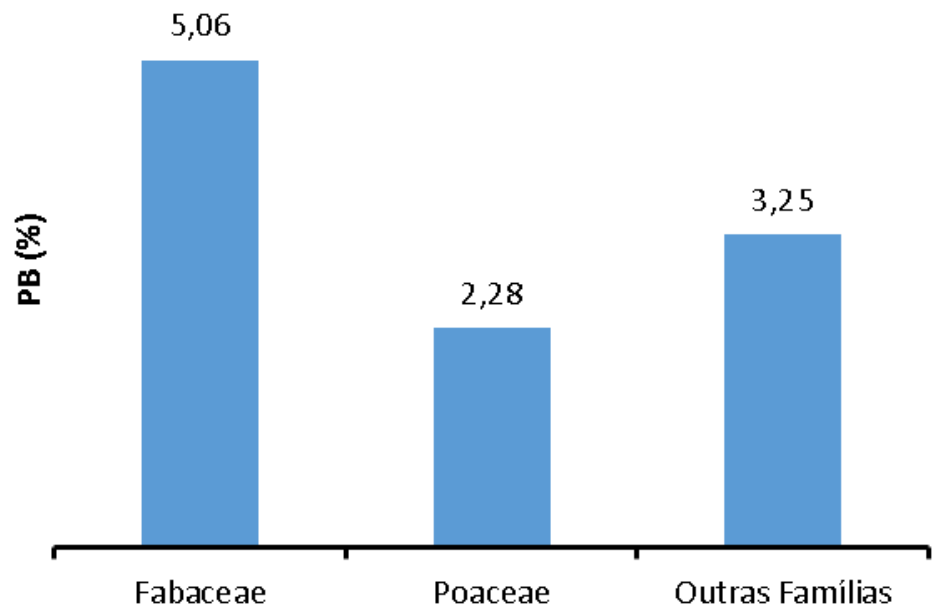

Figura 3. Teor médio de proteína bruta (PB) para as famílias estudadas. 


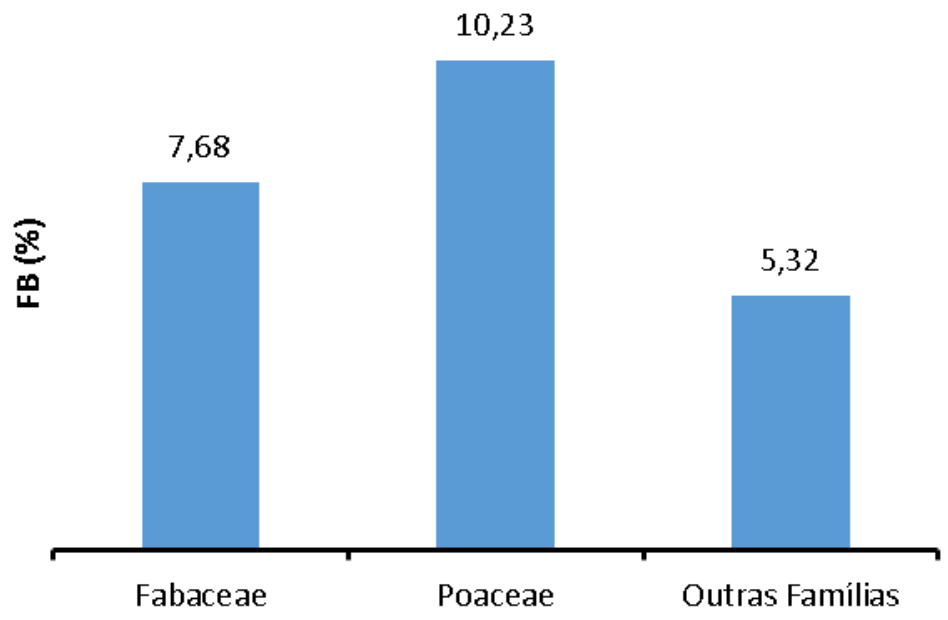

Figura 4. Teor médio de fibra bruta (FB) para as famílias estudadas.

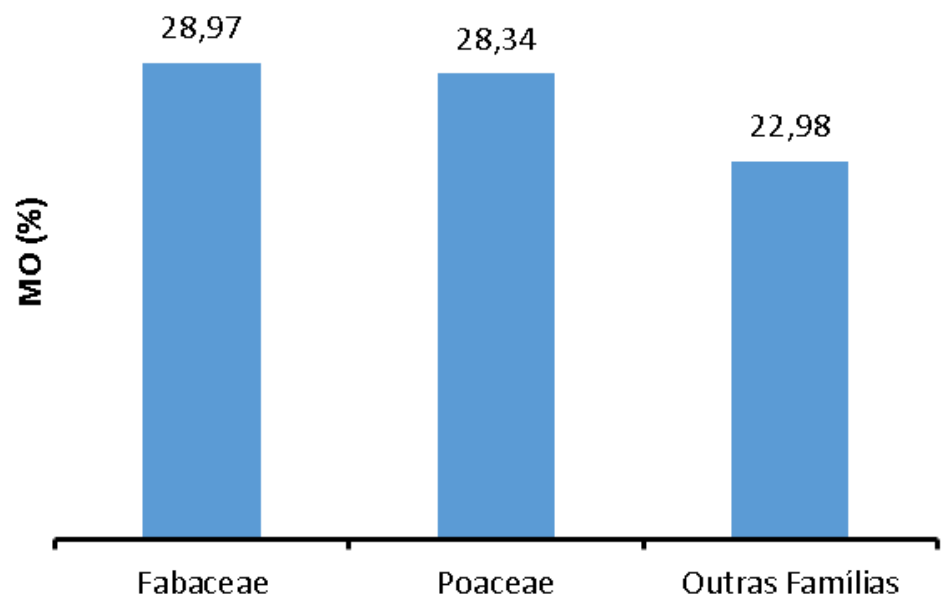

Figura 5. Teor médio de matéria orgânica (MO) para as famílias estudadas.

Os resultados obtidos no presente trabalho se asssemelham aos de Oliveira (2010) com relação à disponibilidade das espécies na fitomassa do componente herbáceo, arbustivo e arbóreo da Caatinga.

\section{Conclusão}

Foram estudadas 176 espécies de plantas em formações herbáceas, arbustivas e arbóreas. Os principais gêneros, em número de espécies, foram Cenchrus, Urochloa e Mimosa. As famílias Poaceae e Fabaceae apresentaram-se como predominantes. Assim sendo, quando associadas à disponibilidade das espécies do componente Outras Famílias, constituem uma fitomassa com grande potencial forrageiro. Constatou-se também uma grande variação na composição bromatológica das espécies estudadas, justificando estudos que promovam a sustentabilidade dos 
sistemas de criação no semiárido brasileiro.

\section{Conflito de interesses}

Os autores declaram não haver conflito de interesses.

\section{Referências}

Andrade, A. P.; Sousa, E. S.; Silva, D. S.; Silva, I. F.; Lima, J. R. S. Produção animal no Bioma Caatinga: paradigmas dos 'pulsos-reservas'. Anais da XLIII Reunião Anual da Sociedade Brasileira de Zootecnia, João Pessoa, 2006.

Araujo Filho, J. A.; Carvalho, F. C.; Garcia, R.; Sousa, R. A. Efeitos da manipulação da vegetação lenhosa sobre a produção e compartimentalização da fitomassa pastável de uma caatinga sucessional. Revista Brasileira de Zootecnia, v. 31, n. 1, p. 11-19, 2002. https://doi.org/10.1590/S1516-3598 2002000100002

Araújo, G. G. L.; Albuquerque, S. G.; Guimarães Filho, C. Opções no uso de forrageiras arbustivo-arbóreas na alimentação animal no semiárido do Nordeste. 2006. Disponível em: <https://ainfo.cnptia. embrapa.br/digital/bitstream/CPATSA/872 3/1/OPB886.pdf>. Acesso em: 18 jan. 2019.

AOAC-Association of Official Analytical Chemistry. Official methods of analysis. 18. ed. Gaithersburg: AOAC, 2010.

Bakke, O. A.; Pereira Filho, J. M.; Bakke, I. A.; Codão, M. A. Produção e utilização da forragem de espécies lenhosas da caatinga. In: Gariglio, M.A.; Sampaio, E. V.; Sá, I. B.; Cestaro, L. A.; Kageyama, P. Y. Uso sustentável e conservação dos recursos florestais da Caatinga. Brasília: Serviço Florestal Brasileiro, 2010. p. 160-179.

Cherney, D. J. R. Forage evaluation in ruminant nutrition. In: Givens, D. I.; Owen, E.; Axford, R. F. E.; Omed, H. M. (Eds.). Characterization of forages by chemical analyses. Wallingford: $\mathrm{CAB}$ International Publishing, 2000. p. 281-300.

Farias, S. G. G.; Rodal M. J. N.; Melo, A. L.; Silva, M. A. M.; Lima, A. L. A. Fisionomia e estrutura de vegetação de Caatinga em diferentes ambientes em Serra Talhada - Pernambuco. Ciência Florestal, v. 26, n. 2, p. 435-448,
2016. https://doi.org/10.5902/19805098 22745

Giulietti, A. M.; Harley, R. M.; Queiroz, L. P.; Wanderley, M. G. L.; Van Den Berg, C. Biodiversidade e conservação das plantas no Brasil. Megadiversidade, v. 1, n. 1, p. 52-61, 2005.

IBGE - Instituto Brasileiro de Geografia e Estatística. Território e Ambiente: Brasil 2017. Rio de Janeiro: IBGE, 2017. Disponível em: <https://cidades.ibge.gov.br/brasil/pb/ panorama>. Acesso em: 18 jan. 2019.

Judd, W. S.; Campbell, C. S.; Kellogg, E. A.; Stevens, P. F.; Donoghue, M. J. Sistemática vegetal: um enfoque filogenético. 3. ed. Porto Alegre: Artmed, 2009.

Martins, T. R. Bromatologia e fundamentos da nutrição. Ribeirão Preto: Universidade do Oeste Paulista, 2007.

Mertens, D. R.; Broderick, G. A.; Simons, R. Efficacy of carbohydrate sources for improving utilization of $\mathrm{N}$ in alfalfa silage. Journal of Dairy Science, v. 77, Suppl. 1, p. 240, 1994.

Mertens, D. R. Physical effective NDF and its use in formulating dairy rations. Anais do II Simpósio Internacional em Bovinos de Leite, Lavras, UFLA-FAEPE, p. 25-36, 2001.

Minson, D.; Stobbs, T. H.; Hegarty, M. P.; Playne, M. J. Measuring the nutritive value of pasture plants. In: Shaw, N. H.; Bryan, W. W. (Eds.). Tropical pasture research: Principles and methods. Wallingford: CAB International Publishing, 1976. p. 308-337.

Moreira, J. N.; Lira, M. A.; Santos, M. V. F.; Ferreira, M. A.; Araújo, G. G. L.; Ferreira, R. L. C.; Silva, G. C. Caracterização da vegetação de Caatinga e da dieta de novilhos no Sertão de Pernambuco. Pesquisa Agropecuaria Brasileira, v. 41, n. 11, p. 1643-1651, 2006. https://doi.org/10.1590/S0100-204X2006 001100011

Mott, G.; Moore, J. E. Forage evaluation techniques in perspective. National Conference on Forage Evaluation and Utilization, Lincoln, Nebraska, Nebraska Center of Continuing Education, p. 1-10, 1970.

Oliveira, F. R. B. Valor nutritivo e consumo de plantas arbóreas, arbustivas e 
herbáceas da Caatinga. Petrolina: UNIVASF, 2010. (Dissertação de mestrado).

Pereira Junior, L. R.; Andrade, A. P.; Araújo, K. D. Composição florística e fitossociológica de um fragmento de Caatinga em Monteiro, PB. Holos, v. 6, p. 73-87, 2012. https://doi.org/ 10.15628/holos.2012.1188

Queiroz, D. S.; Gomide, J.A.; Maria, J. Avaliação da folha e do colmo de topo e base de perfilhos de três gramíneas forrageiras. 1 . Digestibilidade in vitro e composição química. Revista Brasileira de Zootecnia, v. $29, \quad$ p. $1, \quad$ p3-60, 2000. https://doi.org/10.1590/S1516-35982000 000100008

Santana, D. F. Y.; Lira, M. A.; Santos, M, V. F.; Ferreira, M. A.; Silva, M. J. A.; Marques, K. A.; Mello, A. C. L.; Santos, D. C. Caracterização da Caatinga e da dieta de novilhos fistulados, na época chuvosa, no semiárido de Pernambuco. Revista Brasileira de Zootecnia, v. 40, n. 1, p. 69-78, 2011. https://doi.org/10.1590/ S1516-35982011000100010

Santos, G. R. A.; Guim, A.; Santos, M. V. F.; Ferreira, M. A.; Lira, M. A.; Dubeux Júnior, J. C. B.; Silva, M. J. Caracterização do pasto de capim-buffel diferido e da dieta de bovinos, durante o período seco no Sertão de Pernambuco. Revista Brasileira de Zootecnia, v. 34, n. 2, p. 454-463, 2005. https://doi.org/10.1590/S1516-35982005 000200012
Santos, M. V. F.; Lira, M.; Dubeux Junior, J. C. B.; Guim, A.; Mello, A. C. L.; Cunha, M. V. Potential of Caatinga forage plants in ruminant feeding. Revista Brasileira de Zootecnia, v. 39, supl. esp., p. 204-215, 2010. https://doi.org/10.1590/S1516-35982010 001300023

SAS Institute. User's Guide: Statistics. Version 9.2. Cary, USA: North Carolina State University, 2008.

Silva, D. J.; Queiroz, A. C. Análises de alimentos: métodos químicos e biológicos. 3. ed. Viçosa: UFV, 2002.

Van Soest, P. J. Nutritional ecology of the ruminant. 2. ed. Ithaca: Cornell University Press, 1994.

Voltolini, T. V.; Neves, A. L. A.; Guimarães Filho, G.; Nogueira, D. M.; Campeche, D. F. B.; Araújo, G. G. L.; Moreira, J. N.; Veschi, J. L. A.; Santos, R. D.; Morais, S. A. Alternativas alimentares e sistemas de produção animal para o semiárido. In: Sá, I. B.; Silva, P. C. G. (Eds.). Semiárido brasileiro: pesquisa, desenvolvimento e inovação. Petrolina: Embrapa Semiárido, 2010. p. 143-206.

Informação da Licença: Este é um artigo Open Access distribuído sob os termos da Licença Creative Commons Attribution, que permite uso irrestrito, distribuição e reprodução em qualquer meio, desde que a obra original seja devidamente citada. 